Methods Multi-methods comprising two stages: 1) group and individual interviews with S.Asians aged 50-74 years purposively sampled from faith-based venues in Oxfordshire (Mosques, Hindu temples and Sikh Gurdwaras), religious festivals and local community groups for maximum variation. Semistructured interviews based on the Theoretical Domains Framework (TDF) investigated determinants of bowel screening completion. Interviews were recorded, transcribed, and analysed using framework analysis and findings mapped onto the COM-B Behaviour Change Wheel; 2) Co-production of intervention during two workshops with key stakeholders and target population. Findings from stage one were presented, feedback sought and amendments to the intervention prototype were made.

Results To-date 25 adults recruited of Indian, Pakistani and Bangladeshi ethnicity with variation in age, gender, first language, faith, compliance with bowel screening. Key barriers and TDF domains that they mapped to were: - lack of knowledge about bowel cancer and screening; lack of language, literacy and physical ability (skills) to carry out the home test; confidence to carry it out correctly (belief about capabilities); appropriate space and time to carry out the test (environmental context and resources); putting off undertaking the test (memory attention and decision processes); risk perception and fear of cancer (emotions). Enablers were: social influences from peers; goals and motivations. Data collection and workshops will be completed by May 2020 .

Conclusion Early results suggest an intervention comprising education, persuasion, modelling and enablement functions could increase completion of the home test. An intervention prototype will be produced and further funding sought for intervention refinement and evaluation of early feasibility and acceptability.

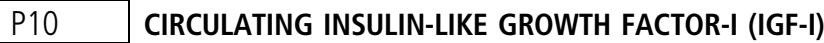 CONCENTRATIONS AND INCIDENCE OF CANCER AT 26 SITES: PROSPECTIVE ANALYSES IN UK BIOBANK}

${ }^{1} \mathrm{~A}$ Knuppel*, ${ }^{1}$ GK Fensom, ${ }^{1} E L$ Watts, ${ }^{2} \mathrm{MJ}$ Gunter, ${ }^{2} \mathrm{~N}$ Murphy, ${ }^{1} \mathrm{~K}$ Papier, ${ }^{1} \mathrm{~A}$ PerezCornago, 'JA Schmidt, ${ }^{3} \mathrm{~K}$ Smith-Byrne, ${ }^{1} \mathrm{TJ}$ Key, ${ }^{1} \mathrm{RC}$ Travis. ${ }^{1}$ Cancer Epidemiology Unit, Nuffield Department of Population Health, University of Oxford, Oxford, UK; ${ }^{2}$ Section of Nutrition and Metabolism, International Agency for Research on Cancer, Lyon, France; ${ }^{3}$ Genetic Epidemiology Group, International Agency for Research on Cancer, Lyon, France

\subsection{6/jech-2020-SSMabstracts. 106}

Background Insulin-like growth factor-I (IGF-I) is suggested to support cancer cell growth and proliferation. Pre-diagnostic circulating IGF-I concentrations have been shown to be positively associated with breast cancer, prostate cancer and colorectal cancer but evidence for less common cancer sites is limited. The aim of this study was to investigate the associations between serum IGF-I concentrations and the incidence of rarer cancers using an outcome-wide approach to study cancers at 26 sites in UK Biobank, in which serum concentrations of IGF-I were measured for $\sim 467,000$ participants (93\%).

Methods We analysed data from 394,406 cancer-free participants (52\% women). IGF-I was measured in serum collected at baseline and in a subsample of 14,149 participants again in repeat samples collected during follow-up. Cancer diagnosis and death due to cancer during follow-up were determined using data-linkage with cancer and death registries. Multivariable-adjusted Cox proportional hazards models were used to determine associations between baseline serum IGF-I concentrations and cancer incidence, using the repeated measurements to correct estimates for regression dilution.

Results After a mean follow-up of 6.9 years, 23,496 participants were diagnosed with a malignant cancer. Higher IGF-I concentration was associated with an increased risk of colorectal cancer (hazard ratio per $5 \mathrm{nmol} / \mathrm{l} 1.10$, 95\%-CI 1.05-1.15), colon cancer $(1.11,1.05-1.17)$, malignant melanoma (1.08, 1.01-1.15), breast cancer in women $(1.11,1.07-1.15)$, prostate cancer $(1.08,1.04-1.11)$, thyroid cancer $(1.23,1.05-1.43)$ and multiple myeloma $(1.13,1.01-1.27)$, and a reduced risk of oral (0.86, 0.77-0.97), liver (0.37, 0.30-0.45), endometrial (0.90, $0.82-1.00)$ and ovarian cancer $(0.88,0.78-0.99)$.

Conclusion Higher IGF-I concentrations were associated with higher risks of cancer at the established sites (breast, colorectal and prostate cancer) and malignant melanoma, thyroid cancer and multiple myeloma. Higher IGF-I concentrations were associated with lower risks of oral, liver, endometrial and ovarian cancer; longer follow-up is needed to investigate the possible role of reverse causality.

\section{P11 IMPROVING PROSTATE CANCER CARE THROUGH THE 'OUTLIER PROCESS': A NATIONAL QUALITY IMPROVEMENT WORKSHOP}

${ }^{1,2}$ J Nossiter* ${ }^{1,2} \mathrm{M}$ Morris, ${ }^{1,2} \mathrm{M}$ Parry, ${ }^{1} \mathrm{~A}$ Sujenthiran, ${ }^{3} \mathrm{~A}$ Aggarwal, ${ }^{4} \mathrm{P}$ Cathcart, ${ }^{5} \mathrm{H}$ Payne, ${ }^{6} \mathrm{~N}$ Clarke, ${ }^{2} \mathrm{~J}$ van der Meulen. ${ }^{1}$ Clinical Effectiveness Unit, Royal College of Surgeons of England, London, UK; ${ }^{2}$ Department of Health Services Research and Policy, London School of Hygiene and tropical Medicine, London, UK; ${ }^{3}$ Department of Cancer Epidemiology, Population and Global Health, Kings' College, London, UK; ${ }^{4}$ Department of Urology, Guy's and St Thomas' NHS Foundation Trust, London, UK; ${ }^{5}$ Department of Oncology, University College London Hospitals, London, UK; ${ }^{6}$ Departments of Urology, Salford Royal and The Christie NHS Foundation Trusts, Manchester, UK

10.1136/jech-2020-SSMabstracts. 107

Background The National Prostate Cancer Audit (NPCA) reports publicly performance indicators for all hospitals in England and Wales providing radical prostate cancer treatment, identifying those with results that fall outside the 'accepted range' as 'potential negative outliers'. Hospitals with outlying results are requested to provide a formal response.

This 'outlier process', targeting a limited number of hospitals, mirrors a 'high-risk approach' of preventing poor quality care in contrast to a 'population approach' that would target all hospitals. We invited clinicians to a national workshop to learn how the outlier process contributes to quality improvement.

Methods The workshop started with presentations on reducing the 'toxicity' of radical prostate cancer treatment. Then, clinicians from three hospitals identified as outliers shared their experience of the process and the changes in practice they had made as a result. We collected data in three ways. First, an online platform was used to gather comments from participants during the workshop. Second, a number of participants were interviewed about the outlier process as a means to improve quality of care. Third, feedback was sought after the workshop from all participants. Responses were collated and analysed for themes.

Results Sixty-nine clinicians attended including urologists, oncologists, radiographers and nurses, representing a spread of hospitals across England and Wales. There were 6 interviews, 21 online comments and 31 responses after the workshop. The clinicians representing outlying hospitals highlighted the 